\title{
Existence and Multiplicity of Solutions for a Biharmonic Equation with $p(x)$-Kirchhoff Type
}

\author{
Qi Zhang and Qing Miao \\ School of Mathematics and Computer Science, Yunnan Minzu University, Kunming, China \\ Correspondence should be addressed to Qing Miao; miaoqing_0404@163.com
}

Received 26 October 2021; Revised 12 November 2021; Accepted 13 November 2021; Published 2 December 2021

Academic Editor: Rodica Luca

Copyright (c) 2021 Qi Zhang and Qing Miao. This is an open access article distributed under the Creative Commons Attribution License, which permits unrestricted use, distribution, and reproduction in any medium, provided the original work is properly cited.

Based on the basic theory and critical point theory of variable exponential Lebesgue Sobolev space, this paper investigates the existence and multiplicity of solutions for a class of nonlocal elliptic equations with Navier boundary value conditions when (AR) condition does not hold and improves or generalizes the original conclusions.

\section{Introduction}

We deal with the following system:

$$
\begin{cases}\left(a+b \int_{\Omega} \frac{|\Delta u|^{p(x)}}{p(x)} \mathrm{d} x\right) \Delta_{p(x)}^{2} u=f(x, u), & x \in \Omega, \\ u=\Delta u=0, & x \in \partial \Omega,\end{cases}
$$

where $\Omega \subset \mathbb{R}^{N}$ is a bounded smooth region. The constants $a$ and $b$ satisfy $a>0$ and $b \geq 0 . \quad p(x) \in C(\bar{\Omega})$, and $1<p^{-}:=\inf _{x \in \Omega} p(x) \leq p^{+}:=\sup _{x \in \Omega} p(x)<+\infty$. The nonlinear term $f(x, u) \in C(\bar{\Omega} \times \mathbb{R}, \mathbb{R})$ satisfies Carathéodory condition. And, $\Delta_{p(x)}^{2} u(x)=\Delta\left(|\Delta u|^{p(x)-2} \Delta u\right)$.

The differential equations with $p(x)$-Laplacian or biharmonic operators are derived from nonlinear elastic mechanics and fluid mechanics. This model can describe the physical phenomenon of "point by point anisotropy." Various methods have extensively studied the problem of differential operators with variable exponents, as shown in $[1-6]$. In $[7,8]$, the authors used changeable nonlinear terms to outline the boundary of the real image, eliminate the possible noise, and solve the problems in image processing. Recently, the question

$$
\begin{cases}-\left(a+b \int_{\Omega}|\nabla u|^{2} \mathrm{~d} x\right) \Delta u=f(x, u), & x \in \Omega, \\ u=0, & x \in \partial \Omega,\end{cases}
$$

has been widely concerned by many scholars after Lions [9] found an abstract method to solve this problem. Furthermore, many interesting results can be seen in [10-12]. In addition, in [13], Dai and Liu studied the following cases with a variable exponent:

$$
\begin{cases}-\left(a+b \int_{\Omega} \frac{1}{p(x)}|\nabla u|^{p(x)} \mathrm{d} x\right) \operatorname{div}\left(\left|\nabla u^{p(x)-2} \nabla u\right|\right)=f(x, u), & x \in \Omega, \\ u=0, & x \in \partial \Omega .\end{cases}
$$


According to the variational method and the basis theorem of Sobolev space under suitable assumptions, the infinite positive solutions of the problem with $p(x)$-Laplace operator is obtained.

As we all know, the Ambrosetti-Rabinowitz [14] (it is written as $(\mathrm{AR}))$ condition can not only ensure that $f(x, s)$ is superlinear with respect to the variable $s$ at infinity but also ensure the boundedness of the Palais-Smale (it is written as (PS)) sequence. Therefore, (AR) condition is essential for the study of many boundary value problems, see [15-18]. In particular, when $a=1$ and $b=0$ in problem (1) and (AR) condition holds, the existence of solution of problem (1) is discussed in [16]. In [17], Guo and Zhao made reasonable presupposition on the nonlinear terms $M$ and $f$, respectively, and obtained the existence number of solutions for $p(x)$-Laplace equation in the whole space by applying the basic theory of weighted function and variable exponent space. In [18], Chung, respectively, applied the minimum principle or critical point theory to discuss the problem when the perturbation term $f$ is sublinear or superlinear at infinity. In addition, based on the critical point theory,
$Z_{2}$-group index theory, and operator equation theory, Shu, Lai, and $\mathrm{Xu}$ [19] constructed a lower semicontinuous convex function $\varphi(x(t), t x n(t-\tau))$ with subdifferentiability and obtained infinite subharmonic periodic solutions of secondorder neutral nonlinear functional differential equations. There are similar articles such as [20].

In [21], Wang and An studied the following problem:

$$
\begin{cases}\Delta^{2} u-M\left(\int_{\Omega}|\nabla u|^{2} \mathrm{~d} x\right) \Delta u=f(x, u), & x \in \Omega, \\ u=\Delta u=0, & x \in \partial \Omega .\end{cases}
$$

According to the mountain pass lemma satisfying (AR) condition, it is proved that the above elliptic equation has at least two solutions, both of which are nontrivial, where one is positive and the other is negative.

However, although many functions satisfy the superlinear growth condition, the (AR) condition does not hold. For example, in [22], Zhang considered the following problems:

$$
\begin{cases}\left(a+b \int_{\Omega} \frac{|\Delta u|^{p(x)}-\lambda|u|^{p(x)}}{p(x)} \mathrm{d} x\right)\left(\Delta_{p(x)}^{2} u-\lambda|u|^{p(x)-2} u\right)=f(x, u), & x \in \Omega, \\ u=\Delta u=0, & x \in \partial \Omega .\end{cases}
$$

When the condition of perturbation term $f$ is weaker than (AR), based on the fountain theorem, it is obtained that there are infinite solutions to the above problem.

In [23], the author further investigated the existence of multiple solutions for formula (1) when the (AR) condition is invalid according to the same critical point theory. It should be noted that although problem (1) has been studied in [23], this paper will prove that under weaker conditions, we can still use the fountain theorem to obtain the same result as that in reference [23], which illustrates that our conclusion is more universal. Furthermore, we will obtain mountain pass solutions and infinitely many solutions converging to 0 .

This article is mainly composed of four sections. Section 2 introduces the basic theory of Lebesgue and Sobolev spaces and gives the related propositions and theorems needed below. Section 3 first proves that the solution of problem (1) exists under reasonable assumptions and then further proves the existence of at least one nontrivial solution by quoting mountain pass lemma. In the last but most important part (Section 4), the sufficient conditions for the existence of multiple solutions of problem (1) are obtained according to the variational method and critical point theory. It is worth noting that the original results are generalized and improved in this part, and we will explain in detail that our results are better later.

\section{Preliminaries}

Let

$$
\begin{aligned}
& C_{+}(\bar{\Omega})=\{q(x) \mid q(x) \in C(\bar{\Omega}), q(x)>1, x \in \Omega\} \\
& \forall q(x) \in C(\bar{\Omega}), q^{+}:=\max _{\bar{\Omega}} q(x), q^{-}:=\min _{\bar{\Omega}} q(x) .
\end{aligned}
$$

Define the following spaces:

$$
L^{p(x)}(\Omega)=\left\{u: \Omega \longrightarrow \mathbb{R} \text { measurable, } \int_{\Omega}|u(x)|^{p(x)} \mathrm{d} x<\infty\right\},
$$

having the norm

$$
|u|_{p(x)}=\inf \left\{\vartheta>0: \int_{\Omega}\left|\frac{u(x)}{\vartheta}\right|^{p(x)} \mathrm{d} x \leq 1\right\} .
$$

In addition, the variable exponential Sobolev space is defined as follows:

$$
W^{h, p(x)}(\Omega)=\left\{u \in L^{p(x)}(\Omega): D^{\sigma} u \in L^{p(x)}(\Omega),|\sigma| \leq h, h \in Z_{+}\right\},
$$

having the norm

$$
|u|_{h, p(x)}=\sum_{|\sigma| \leq h}\left|D^{\sigma} u\right|_{p(x)},
$$


where $\sigma=\left(\sigma_{1}, \sigma_{2}, \ldots, \sigma_{n}\right)$ and formula $|\sigma|=\sum_{j=1}^{n} \sigma_{j}$ is true. The closure of $C_{0}^{\infty}(\Omega)$ in $W^{h, p(x)}(\Omega)$ is the $W_{0}^{h, p(x)}(\Omega)$. Besides, from [24], we know that $L^{p(x)}(\Omega)$ and $W^{h, p(x)}(\Omega)$ are Banach space and satisfy separability, uniform convexity, and reflexivity.

Denote $X=W_{0}^{1, p(x)}(\Omega) \cap W^{2, p(x)}(\Omega)$, which is a reflexive and separable Banach space endowed with the norm

$$
\|u\|=\|u\|_{X}=\|u\|_{1, p(x)}+\|u\|_{2, p(x)} .
$$

We have

$$
\|u\|=\inf \left\{\kappa>0: \int_{\Omega}\left|\frac{\Delta u(x)}{\kappa}\right|^{p(x)} \mathrm{d} x \leq 1\right\} .
$$

By [25], we realize that $\|\cdot\|_{2, p(\cdot)},|\Delta \cdot|_{p(\cdot)}$, and $\|\cdot\|$ are equivalent to the norm of $X$.

Define the energy functional $\mathrm{H}: X \longrightarrow \mathbb{R}$ as follows:

$$
\begin{aligned}
\mathrm{H}(u)= & a \int_{\Omega} \frac{|\Delta u|^{p(x)}}{p(x)} \mathrm{d} x+\frac{b}{2}\left(\int_{\Omega} \frac{|\Delta u|^{p(x)}}{p(x)} \mathrm{d} x\right)^{2} \\
& -\int_{\Omega} F(x, u) \mathrm{d} x,
\end{aligned}
$$

where $F(x, u)=\int_{0}^{u} f(x, t) \mathrm{d} t$ and $\mathrm{H} \in C^{1}(X, \mathbb{R})$. Furthermore, $\forall u, v \in X$, we know that

$$
\begin{aligned}
\left\langle\mathrm{H}^{\prime}(u), v\right\rangle= & \left(a+b \int_{\Omega} \frac{|\Delta u|^{p(x)}}{p(x)} \mathrm{d} x\right) \int_{\Omega}|\Delta u|^{p(x)-2} \Delta u \Delta v \mathrm{~d} x \\
& -\int_{\Omega} f(x, u) v \mathrm{~d} x .
\end{aligned}
$$

Above all, it is easy to obtain that if $u \in X$ is the weak solution of problem (1), it means that $u$ is the critical point of functional $\mathrm{H}$.

Proposition 1 (see $[2,23])$. Assume that $\partial \Omega$ possesses the cone property; moreover, $\forall x \in \bar{\Omega}, \quad q(x) \in C_{+}(\bar{\Omega})$ with $q(x)<p_{2}^{*}(x)$; then, $X \longrightarrow L^{q(x)}(\Omega)$ is a continuous and compact embedding, where

$$
p_{2}^{*}(x)= \begin{cases}\frac{N p(x)}{N-2 p(x)}, & p(x)<\frac{N}{2}, \\ \infty, & p(x) \geq \frac{N}{2} .\end{cases}
$$

Proposition 2 (see [1]).

(1) $J$ is a homeomorphism.

(2) J: $X \longrightarrow X^{*}$ is a strictly monotone, bounded, and continuous operator.

(3) $J$ is a mapping of type $\left(S_{+}\right)$, namely, on $X$; if $u_{n} \longrightarrow^{w} u$ and, furthermore, $\lim _{n \longrightarrow \infty}\left(J\left(u_{n}\right)-\right.$ $\left.J(u), u_{n}-u\right) \leq 0$, then $u_{n} \longrightarrow u$ in $X$.
Remark 1. According to Proposition 2, we have that $\mathrm{H}$ satisfies type $\left(S_{+}\right)$and weakly lower semicontinuous.

Proposition 3 (see [24]). Assume $\left(1 / p_{1}(x)\right)+\left(1 / p_{2}\right.$ $(x))=1$; then, $\forall u \in L^{p_{1}(x)}(\Omega)$ and $\forall v \in L^{p_{2}(x)}(\Omega)$, we have

$$
\left|\int_{\Omega} u v \mathrm{~d} x\right| \leq\left(\frac{1}{p_{1}^{-}}+\frac{1}{p_{2}^{-}}\right)|u|_{p_{1}(x)}|v|_{p_{2}(x)},
$$

where $L^{p_{1}(x)}(\Omega)$ and $L^{p_{2}(x)}(\Omega)$ are conjugate space.

Proposition 4 (see $[2,24]$ ). Let $J(u)=\int_{\Omega}|\Delta u|^{p(x)} d x$; $\forall u \in W^{2, p(x)}(\Omega) \cap W_{0}^{1, p(x)}(\Omega)$, we deduce

$$
\begin{aligned}
& (h 1)\|u\|<1(=1,>1) \Longleftrightarrow J(u)<1(=1,>1), \\
& (h 2)\|u\| \geq 1 \Longrightarrow\|u\|^{p^{-}} \leq J(u) \leq\|u\|^{p^{+}}, \\
& (h 3)\|u\| \leq 1 \Longrightarrow\|u\|^{p^{+}} \leq J(u) \leq\|u\|^{p^{-}}, \\
& (h 4)\|u\| \longrightarrow 0 \Longleftrightarrow J(u) \longrightarrow 0 .
\end{aligned}
$$

Theorem 1 (see [26]). Let $X$ be a real Banach space. If $\Phi \in C^{1}(X, \mathbb{R})$ satisfies (PS) condition and

$$
\begin{aligned}
(Q 1) \Phi(0) & =0, \\
\text { (Q2) } \exists \zeta, \tau>0 \text { such that } \Phi(u) \geq \tau \text { as }\|u\| & =\zeta, \\
\text { (Q3) } \exists v \in X,\|v\|>\zeta \text { such that } \Phi(v) & \leq 0,
\end{aligned}
$$

then $\Phi$ has a critical value expressed as $c=\inf _{l \in \Gamma} \max _{0 \leq t \leq 1} \Phi$ $(l(t))$ with $c \geq \tau$, where $\Gamma:=\{l \in C[0,1] \mid l(0)=0, l(1)=v\}$.

Owing to $X$ as a reflexive and separable Banach space, then there exists $\left\{e_{i}\right\} \subset X$ such that

$$
\begin{aligned}
X_{i} & =\operatorname{span}\left\{e_{i}\right\}, \\
Y_{k} & =\stackrel{\oplus}{\oplus} X_{i=1}, \\
Z_{k} & =\overbrace{i \geq k}^{\infty} X_{i}
\end{aligned}
$$

Thus, $X=Y_{k} \oplus Z_{k}$. If $\bar{k} \in \mathbb{N}$, it is clear that $Y_{\bar{k}}=\oplus_{i=1}^{\bar{k}} X_{i}$ is the $\bar{k}$-dimensional subspace of $X$.

Theorem 2 (see [26]). Assume the following:

(M1) $X$ is a Banach space, $\Psi \in C^{1}(X, \mathbb{R})$ satisfies $\Psi(0)=0$, and $\Psi(-u)=\Psi(u)$. If for each $k=1,2, \ldots$, there exist $\rho_{k}>\lambda_{k}>0$ such that we have the following: (M2) $b_{k}:=\inf _{u \in Z_{k},\|u\|=\lambda_{k}} \Psi(u) \longrightarrow+\infty$ as $k \longrightarrow+\infty$ (M3) $a_{k}:=\max _{u \in Y_{k},\|u\|=\rho_{k}} \Psi(u) \leq 0$

(M4) The functional $\Psi$ satisfies Cerami condition ( $a b$ breviated as $(C)$ condition), namely, for any $\left\{u_{n}\right\} \subset X$ such that $\Psi\left(u_{n}\right) \longrightarrow C$ and $\left\|\Psi^{\prime}\left(u_{n}\right)\right\|\left(1+\left\|u_{n}\right\|\right) \longrightarrow 0$ asn $\longrightarrow+\infty$ has a convergent subsequence, then $\Psi$ has a series of critical values that tend to be positive infinity.

Theorem 3 (see [26]). Assume that (M1) is satisfied and there is $k_{0}>0$ such that, for each $k \geq k_{0}$, there exists $\bar{\rho}_{k}>\bar{\lambda}_{k}>0$ such that we have the following: 
(N1) $\inf _{u \in Z_{k},\|u\|=\bar{\rho}_{k}} \Psi(u) \geq 0$

(N2) $m_{k}:=\max _{u \in Y_{k},\|u\|=\bar{\lambda}_{k}} \Psi(u)<0$

(N3) $n_{k}:=\inf _{u \in Z_{k},\|u\| \leq \bar{\rho}_{k}} \Psi(u) \longrightarrow 0$ as $k \longrightarrow+\infty$

(N4) For every $c \in\left[d_{k_{0}}, 0\right), \Psi$ satisfies $(P S)_{c}^{*}$ condition, i.e., if any sequence $\left\{u_{n_{j}}\right\} \subset X$ such that $n_{j} \longrightarrow+\infty$, $u_{n_{j}} \in Y_{n_{j}}, \Psi\left(u_{n_{j}}\right) \longrightarrow c$, and $\left(\left.\Psi\right|_{Y_{n_{j}}}\right)^{\prime}\left(u_{n_{j}}\right) \longrightarrow 0$ contain a subsequence converging to a critical point of $\Psi$, then $\Psi$ has a series of critical values that converge to zero, where these critical values are negative

Next, the nonlinear term $f(x, t)$ always has to verify the following assumptions.

(g0) $\forall x \in \Omega, t \in \mathbb{R}$, we get

$$
|f(x, t)| \leq c_{1}\left(1+|t|^{\alpha(x)-1}\right),
$$

where $\alpha(x) \in C_{+}(\bar{\Omega})$ and $2 p^{+}<\alpha^{-} \leq \alpha(x) \leq \alpha^{+}<p_{2}^{*}(x)$.

Remark 2. From Proposition 1, we can infer that $X$ is compact-embedded into $L^{p^{-}}(\Omega)$ and $L^{p^{+}}(\Omega)$, respectively, that is to say, for any $u \in X$, there are $r, \bar{c}>0$ such that $|u|_{L^{p^{-}}(\Omega)}^{p^{-}} \leq r\|u\|^{p^{-}}$and $|u|_{L^{p^{+}(\Omega)}}^{p^{+}} \leq \bar{c}\|u\|^{p^{+}}$. Besides, let $\varepsilon>0$ be small enough such that $\varepsilon \bar{c} \leq 2 a / 3 p^{+}$. We have the following:

(g1) There exists $0<\mu<a / r$ such that

$$
\lim _{|t| \longrightarrow \infty} \inf \frac{t f(x, t)-2 p^{+} F(x, t)}{|t|^{p^{-}}}>-\mu \text { uniformly in } x \in \Omega \text {. }
$$

(g2) $\lim _{|t| \longrightarrow \infty} \inf F(x, t) /|t|^{2 p^{+}}=+\infty$ uniformly in $x \in \Omega$

(g3) $f(x, t)=o\left(|t|^{p^{+}-1}\right), t \longrightarrow 0$ uniformly in $x \in \Omega$

(g4) $F(x, t)=F(x,-t), \forall x \in \Omega$ and $t \in \mathbb{R}$

(g5) $f(x, t) \geq c_{2}|t|^{\gamma(x)-1}, t \longrightarrow 0$, where $\gamma(x) \in C_{+}(\bar{\Omega})$, $\gamma^{-} \leq \gamma^{+}<p^{-}$for a.e. $x \in \Omega$

The main conclusions and proofs of the article will be introduced in the following. Note that $c_{i}(i=1,2, \ldots$,$) are$ positive constants and have different meanings in different places.

\section{Existence of Solutions}

Theorem 4. Assume

$$
|f(x, t)| \leq c_{3}\left(1+|t|^{\beta-1}\right),
$$

where $1 \leq \beta<p^{-}<p_{2}^{*}$; then, there is a weak solution to formula (1).

Proof. By (22), we deduce that $|F(x, t)| \leq c_{4}\left(|t|+|t|^{\beta}\right)$. If $\|u\|$ is large enough and using Proposition 1, we get

$$
\begin{aligned}
\mathrm{H}(u) & =a \int_{\Omega} \frac{|\Delta u|^{p(x)}}{p(x)} \mathrm{d} x+\frac{b}{2}\left(\int_{\Omega} \frac{|\Delta u|^{p(x)}}{p(x)} \mathrm{d} x\right)^{2}-\int_{\Omega} F(x, u) \mathrm{d} x \\
& \geq \frac{a}{p^{+}} \int_{\Omega}|\Delta u|^{p(x)} \mathrm{d} x+\frac{b}{2\left(p^{+}\right)^{2}}\left(\int_{\Omega}|\Delta u|^{p(x)} \mathrm{d} x\right)^{2}-c_{4} \int_{\Omega}|u| \mathrm{d} x-c_{4} \int_{\Omega}|u|^{\beta} \mathrm{d} x \\
& \geq \frac{a}{p^{+}}\|u\|^{p^{-}}+\frac{b}{2\left(p^{+}\right)^{2}}\|u\|^{2 p^{-}}-c_{4}|u|_{L^{1}(\Omega)}-c_{4}|u|_{L^{\beta}(\Omega)}^{\beta} \\
& \geq \frac{a}{p^{+}}\|u\|^{p^{-}}+\frac{b}{2\left(p^{+}\right)^{2}}\|u\|^{2 p^{-}}-c_{5}\|u\|-c_{6}\|u\|^{\beta} .
\end{aligned}
$$

Thanks to $\|u\|>1$, we deduce that

$$
\begin{aligned}
\mathrm{H}(u) & \geq \frac{a}{p^{+}}\|u\|^{p^{-}}+\frac{b}{2\left(p^{+}\right)^{2}}\|u\|^{p^{-}}-c_{5}\|u\|-c_{6}\|u\|^{\beta} \\
& \geq L_{1}\|u\|^{p^{-}}-c_{5}\|u\|-c_{6}\|u\|^{\beta} \\
& \longrightarrow+\infty
\end{aligned}
$$

where $L_{1}=\min \left\{a / p^{+}, b / 2\left(p^{+}\right)^{2}\right\}$. Hence, $\mathrm{H}$ is coercive. Since $X$ is reflexive and $H$ is weakly lower semicontinuous, then $\mathrm{H}$ has a minimum point $u$ in $X$; moreover, $u$ is a weak solution of (1).
Lemma 1. If $f$ satisfies $(g 0)$ and $(g 1)$, then $\mathrm{H}$ satisfies $(C)$ condition.

Proof. Let $\left\{u_{n}\right\} \subset X$ be a Cerami sequence, i.e., $\mathrm{H}\left(u_{n}\right) \longrightarrow C$ and $\left\|\mathrm{H}^{\prime}\left(u_{n}\right)\right\|\left(1+\left\|u_{n}\right\|\right) \longrightarrow 0$. Next, we show that $\left\{u_{n}\right\}$ is bounded in $X$. Assume $\left\|u_{n}\right\| \longrightarrow+\infty$ as $n \longrightarrow+\infty$; by (g1), there exists $R>1$ such that

$$
t f(x, t)-2 p^{+} F(x, t) \geq-\mu|t|^{p^{-}}, \quad \forall|t|>R .
$$
that 


$$
\begin{aligned}
C+\left\|u_{n}\right\| & \geq \mathrm{H}\left(u_{n}\right)+\frac{1}{2 p^{+}}\left\|\mathrm{H}^{\prime}\left(u_{n}\right)\right\|\left(1+\left\|u_{n}\right\|\right) \geq \mathrm{H}\left(u_{n}\right)-\frac{1}{2 p^{+}}\left\langle\mathrm{H}^{\prime}\left(u_{n}\right), u_{n}\right\rangle \\
& \geq \frac{a}{2 p^{+}} \int_{\Omega}\left|\Delta u_{n}\right|^{p(x)} \mathrm{d} x+\int_{\Omega} \frac{1}{2 p^{+}}\left(f\left(x, u_{n}\right) u_{n}-F\left(x, u_{n}\right)\right) \mathrm{d} x \\
& =\frac{a}{2 p^{+}} \int_{\Omega}\left|\Delta u_{n}\right|^{p(x)} \mathrm{d} x+\frac{1}{2 p^{+}}\left[\left(\int_{\Omega_{n}}+\int_{\Omega / \Omega_{n}}\right)\left(f\left(x, u_{n}\right) u_{n}-2 p^{+} F\left(x, u_{n}\right)\right) \mathrm{d} x\right] \\
& \geq \frac{a}{2 p^{+}}\left\|u_{n}\right\|^{p^{-}}-\frac{\mu}{2 p^{+}} \int_{\Omega_{n}}\left|u_{n}\right|^{p^{-}} \mathrm{d} x-\delta \\
& \geq \frac{a}{2 p^{+}}\left\|u_{n}\right\|^{p^{-}}-\frac{\mu r}{2 p^{+}}\left\|u_{n}\right\|^{p^{-}}-\delta \\
& =\frac{a-\mu r}{2 p^{+}}\left\|u_{n}\right\|^{p^{-}}-\delta,
\end{aligned}
$$

since $p^{-}>1$ and $\mu<a / r$; so, this is contradictory, which means $\left\{u_{n}\right\}$ is bounded. Without loss of generality, if $u_{n} \longrightarrow^{w} u$, we have $\mathrm{H}^{\prime}\left(u_{n}\right)\left(u_{n}-u\right) \longrightarrow 0$. Then, it can be obtained $u_{n} \longrightarrow u$ according to conditions (g0), Hölder inequality, and Remark 1, and the specific proof can be seen in [23].

Remark 3. This is the same as the proof of Lemma 1, and it can be obtained that $\mathrm{H}$ also satisfies the (PS) sequence.
Theorem 5. If $f(x, t)$ satisfies $(g 0),(g 1),(g 2)$, and $(g 3)$, then there is a nontrivial solution to problem (1).

Proof. Obviously, $\mathrm{H}$ satisfies (PS) sequence and $\mathrm{H}(0)=0$. Next, we need to show (Q2) and (Q3).

First of all, (Q2) is established. From (g0) and (g3), we can infer that

$$
F(x, t) \leq \varepsilon|t|^{p^{+}}+c_{7}|t|^{\alpha(x)}, \quad \forall(x, t) \in \Omega \times \mathbb{R} .
$$

When $\|u\| \leq 1$, we have

$$
\begin{aligned}
\mathrm{H}(u) & \geq \frac{a}{p^{+}} \int_{\Omega}|\Delta u|^{p(x)} \mathrm{d} x+\frac{b}{2\left(p^{+}\right)^{2}}\left(\int_{\Omega}|\Delta u|^{p(x)} \mathrm{d} x\right)^{2}-\varepsilon \int_{\Omega}|u|^{p^{+}} \mathrm{d} x-c_{7} \int_{\Omega}|u|^{\alpha(x)} \mathrm{d} x \\
& \geq \frac{a}{p^{+}}\|u\|^{p^{+}}+\frac{b}{2\left(p^{+}\right)^{2}}\|u\|^{2 p^{+}}-\varepsilon \bar{c}\|u\|^{p^{+}}-c_{8}\|u\|^{\alpha^{-}} \\
& \geq \frac{a}{3 p^{+}}\|u\|^{p^{+}}+\frac{b}{2\left(p^{+}\right)^{2}}\|u\|^{2 p^{+}}-c_{8}\|u\|^{\alpha^{-}} \\
& \geq L_{2}\|u\|^{2 p^{+}}-c_{8}\|u\|^{\alpha^{-}}
\end{aligned}
$$

where $L_{2}=\min \left\{a / 3 p^{+}, b / 2\left(p^{+}\right)^{2}\right\}$; therefore, there exist $\zeta>0$ and $\tau>0$ such that $\mathrm{H}(u) \geq \tau>0$ for every $\|u\|=\zeta$.

It only remains to prove (Q3). By (g2), we obtain that

$$
F(x, t) \geq \eta|t|^{2 p^{+}}-\widetilde{c},
$$

$\forall w \in X \backslash\{0\}$ with $\|w\|=1$ and $t>1$; we gain $\forall \eta>0$, there exists $\widetilde{c}>0$ that satisfies

$$
\begin{aligned}
\mathrm{H}(t w) & \leq \frac{a t^{p^{+}}}{p^{-}} \int_{\Omega}|\Delta w|^{p(x)} \mathrm{d} x+\frac{b t^{2 p^{+}}}{2\left(p^{-}\right)^{2}}\left(\int_{\Omega}|\Delta w|^{p(x)} \mathrm{d} x\right)^{2}-\eta \int_{\Omega}|t w|^{2 p^{+}} \mathrm{d} x+\widetilde{c}|\Omega| \\
& \leq t^{2 p^{+}}\left[\frac{a}{p^{-}}\|w\|^{p^{+}}+\frac{b}{2\left(p^{-}\right)^{2}}\|w\|^{2 p^{+}}\right]-\eta t^{2 p^{+}} \int_{\Omega}|w|^{2 p^{+}} \mathrm{d} x+\widetilde{c}|\Omega| \\
& \leq\left(2 L_{3}-\eta \widetilde{C}\right) t^{2 p^{+}}+\widetilde{c}|\Omega|,
\end{aligned}
$$


where $L_{3}=\max \left\{a / p^{-}, b / 2\left(p^{-}\right)^{2}\right\}$ and $\widetilde{C}$ is a normal number. If $\eta$ is sufficiently large and $t \longrightarrow+\infty$, then $\mathrm{H}(t w) \longrightarrow-\infty$. Hence, based on Theorem 1, we can get that $\mathrm{H}$ has at least one nontrivial solution.

\section{Multiple Solutions}

The conclusions of this section are as follows.

Theorem 6. If $f$ satisfies ( $g 0),(g 1),(g 2)$, and (g4), then equation (1) has a sequence of solutions $\left\{ \pm u_{k}\right\}_{k=1}^{\infty}$ such that $\mathrm{H}\left( \pm u_{k}\right) \longrightarrow+\infty$ ask $\longrightarrow+\infty$.

Theorem 7. If satisfies (g0), (g1), (g3), (g4), and (g5), then equation (1) has a sequence of solutions $\left\{ \pm v_{k}\right\}_{k=1}^{\infty}$ such that $\mathrm{H}\left( \pm v_{k}\right)<0$ and $\mathrm{H}\left( \pm v_{k}\right) \longrightarrow 0$ as $k \longrightarrow+\infty$.

Next, we will use the fountain theorem and dual fountain theorem to prove Theorem 6 and Theorem 7, respectively.
Lemma 2 (see $[2,18]$ ). If $\alpha(x) \in C_{+}(\bar{\Omega})$ and $\alpha(x)<p_{2}^{*}(x)$ for any $x \in \bar{\Omega}$, denote

$$
\xi_{k}=\sup \left\{|v|_{\alpha(x)}:\|v\|=1, v \in Z_{k}\right\},
$$$$
\text { then } \lim _{k \longrightarrow+\infty} \xi_{k}=0 \text {. }
$$

Lemma 3 (see $[2,18]$ ). If conditions ( $g 0)$ and ( $g 1)$ are true, then $\mathrm{H}$ satisfies the $(P S)_{c}^{*}$ condition.

Proof of Theorem 6. Thanks to Lemma 1 and (g4), it is easy to gain (M1) and (M4). Now, we only need to prove that (M2) and (M3) are tenable.

Let us start with (M2). $\forall u \in Z_{k},\|u\|=\lambda_{k}=$ $\left(4 c_{11} \xi_{k}^{\alpha^{+}} L_{1}^{-1}\right)^{1 / p^{-}-\alpha^{+}}$; by (g0), we have

$$
\begin{aligned}
\mathrm{H}(u) & \geq \frac{a}{p^{+}} \int_{\Omega}|\Delta u|^{p(x)} \mathrm{d} x+\frac{b}{2\left(p^{+}\right)^{2}}\left(\int_{\Omega}|\Delta u|^{p(x)} \mathrm{d} x\right)^{2}-c_{9} \int_{\Omega}|u|^{\alpha(x)} \mathrm{d} x-c_{9} \int_{\Omega}|u| \mathrm{d} x \\
& \geq \frac{a}{p^{+}}\|u\|^{p^{-}}+\frac{b}{2\left(p^{+}\right)^{2}}\|u\|^{2 p^{-}}-c_{9} \int_{\Omega}|u|^{\alpha(x)} \mathrm{d} x-c_{10}\|u\| \\
& \geq L_{1}\|u\|^{p^{-}}-c_{10}\|u\|- \begin{cases}c_{9}, & |u|_{\alpha(x)} \leq 1, \\
c_{11}\left(\xi_{k}\|u\|\right)^{\alpha^{+}}, & |u|_{\alpha(x)}>1,\end{cases} \\
& \geq L_{1}\|u\|^{p^{-}}-c_{11} \xi_{k}^{\alpha^{+}}\|u\|^{\alpha^{+}}-c_{10}\|u\|-c_{11},
\end{aligned}
$$

and we know $\xi_{k} \longrightarrow 0$ and $\lambda_{k} \longrightarrow+\infty$ as $k \longrightarrow+\infty$ after that

$$
\begin{aligned}
& \mathrm{H}(u) \geq \frac{3}{4} L_{1}\|u\|^{p^{-}}-c_{10}\|u\|-c_{11} \\
& \text { since } p^{-}>1, \text { so, } b_{k} \\
&:=\inf _{u \in Z_{k},\|u\|=\lambda_{k}} \mathrm{H}(u) \longrightarrow+\infty \text {, as } k \longrightarrow+\infty .
\end{aligned}
$$

Then, we show the correctness of (M3). By (29), we infer that for any $u \in Y_{k}$, with $\|u\| \geq 1$, afterwards

$$
\begin{aligned}
\mathrm{H}(u) & \leq \frac{a}{p^{-}}\|u\|^{p^{+}}+\frac{b}{2\left(p^{-}\right)^{2}}\|u\|^{2 p^{+}}-\eta \int_{\Omega}|u|^{2 p^{+}} \mathrm{d} x+\widetilde{c}|\Omega| \\
& \leq 2 L_{3}\|u\|^{2 p^{+}}-\eta \int_{\Omega}|u|^{2 p^{+}} \mathrm{d} x+\widetilde{c}|\Omega| .
\end{aligned}
$$

Since $\operatorname{dim} Y_{k}<+\infty$ and the norm in a finite-dimensional space is equivalent, when $\eta$ is sufficiently large and $\|u\| \longrightarrow+\infty$, we know that $\mathrm{H}(u) \longrightarrow-\infty$.

Therefore, the proof of Theorem 6 is finished.
Remark 4. When (g0), (g2), and (g4) are established, the author of [23] has studied the multiplicity of solutions to equation (1) and assumed that

$$
\begin{aligned}
(g 1)^{\prime} \exists R & >0 \text { such that } \mathscr{F}(x, t):=t f(x, t)-2 p^{+} F(x, t) \\
& \geq 0 \text { for all } x \in \Omega,|t| \geq R .
\end{aligned}
$$

However,

$$
t f(x, t)-2 p^{+} F(x, t) \geq 0 \geq-\mu|t|^{p^{-}}, \quad \forall|t|>R .
$$

Therefore, condition ( $g 1)$ is weaker than condition $(g 1)^{\prime}$, which shows that we generalize the original results and our results are better. In other words, our conclusion is more universal.

Proof of Theorem 7. By (g4) and Lemma 3, we will deduce that H satisfies (M1) and (N4). Next step, all we have to do is check (N1)-(N3).

The first step is to prove (N1). Due to Lemma 2 and (27), for $k \geq k_{0}$ and $u \in Z_{k} \cap T_{1}$, with $T_{1}=\left\{u \mid u \in Z_{k}, 0<u<1\right.$, $\left.\|u\|=\bar{\rho}_{k}=1\right\}$, we deduce that 


$$
\begin{aligned}
\mathrm{H}(u) & \geq \frac{a}{p^{+}} \int_{\Omega}|\Delta u|^{p(x)} \mathrm{d} x+\frac{b}{2\left(p^{+}\right)^{2}}\left(\int_{\Omega}|\Delta u|^{p(x)} \mathrm{d} x\right)^{2}-\varepsilon \int_{\Omega}|u|^{p^{+}} \mathrm{d} x-c_{7} \int_{\Omega}|u|^{\alpha(x)} \mathrm{d} x \\
& \geq \frac{a}{p^{+}}\|u\|^{p^{+}}+\frac{b}{2\left(p^{+}\right)^{2}}\|u\|^{2 p^{+}}-\varepsilon \bar{c}\|u\|^{p^{+}}- \begin{cases}c_{7}\left(\xi_{k}\|u\|\right)^{\alpha^{-}}, & |u|_{\alpha(x)} \leq 1, \\
c_{7}\left(\xi_{k}\|u\|\right)^{\alpha^{+}}, & |u|_{\alpha(x)}>1,\end{cases} \\
& \geq\left(\frac{a}{3 p^{+}}+\frac{b}{2\left(p^{+}\right)^{2}}\right)\|u\|^{2 p^{+}}- \begin{cases}c_{7} \xi_{k}^{\alpha^{-}}\|u\|^{\alpha^{-}}, & |u|_{\alpha(x)} \leq 1, \\
c_{7} \xi_{k}^{\alpha^{+}}\|u\|^{\alpha^{+}}, & |u|_{\alpha(x)}>1,\end{cases} \\
& \geq L_{2}-\left\{\begin{array}{l}
c_{7} \xi_{k}^{\alpha^{-}}, \quad|u|_{\alpha(x)} \leq 1, \\
c_{7} \xi_{k}^{\alpha^{+}}, \quad|u|_{\alpha(x)}>1,
\end{array}\right.
\end{aligned}
$$

since $\xi_{k} \longrightarrow 0$ belongs to $k \longrightarrow+\infty$; thus, it is clear that $\mathrm{H}(u) \geq L_{2} \geq 0$. That is to say, (N1) was established.
Then, we verify that (N2) is true. $\forall u \in Y_{k}$ and $\|u\|$ are small enough, by (g5), we have

$$
\begin{aligned}
& \mathrm{H}(u) \leq \frac{a}{p^{-}}\|u\|^{p^{-}}+\frac{b}{2\left(p^{-}\right)^{2}}\|u\|^{2 p^{-}}-c_{12} \int_{\Omega}|u|^{\gamma(x)} \mathrm{d} x \\
& \leq 2 L_{3}\|u\|^{p^{-}}-c_{12} \int_{\Omega}|u|^{\gamma(x)} \mathrm{d} x, \\
& \text { due to } \operatorname{dim} Y_{k}<+\infty p^{-}>\gamma^{+} \text {thus } m_{k}:=\max _{u \in Y_{k},\|u\|=\bar{\lambda}_{k}} \mathrm{H}(u)<0 \text {. }
\end{aligned}
$$

Now, it only remains to show (N3). Since $Y_{k} \cap Z_{k} \neq \phi$ and $\bar{\lambda}_{k}<\bar{\rho}_{k}$, then

$$
n_{k}:=\inf _{u \in Z_{k},\|u\| \leq \bar{\rho}_{k}} \mathrm{H}(u) \leq m_{k}:=\max _{u \in Y_{k},\|u\|=\bar{\lambda}_{k}} \mathrm{H}(u)<0 .
$$

From (37), $\forall u \in Z_{k} \cap T_{1}$, we gain

$$
\mathrm{H}(u) \geq L_{2}-\left\{\begin{array}{ll}
c_{7} \xi_{k}^{\alpha^{-}}, & |u|_{\alpha(x)} \leq 1, \\
c_{7} \xi_{k}^{\alpha^{+}}, & |u|_{\alpha(x)}>1,
\end{array} \geq- \begin{cases}c_{7} \xi_{k}^{\alpha^{-}}, & |u|_{\alpha(x)} \leq 1, \\
c_{7} \xi_{k}^{\alpha^{+}}, & |u|_{\alpha(x)}>1\end{cases}\right.
$$

noting that $\xi_{k} \longrightarrow 0$ as $k \longrightarrow+\infty$; hence, $n_{k} \longrightarrow 0$, i.e., (N3) is satisfied. Consequently, the Theorem 7 is proved.

\section{Data Availability}

No data were used to support this study.

\section{Conflicts of Interest}

The authors declare that they have no conflicts of interest.

\section{Authors' Contributions}

Each part of this paper is the result of the joint efforts of QZ and QM. They contributed equally to the final version of the paper. All the authors have read and approved the final manuscript.

\section{Acknowledgments}

This project supported by the National Natural Science Foundation of China (No. 11861078) and Scientific Research Foundation of Yunnan Education Department (No. 2021Y661 and No. 2019J0689).

\section{References}

[1] K. Kefi, K. Saoudi, and M. M. AI-Shomrani, "On a kirchhoff singular $p(x)$-biharmonic problem with navier boundary conditions," Acta Applicandae Mathematicae, vol. 170, no. 1, pp. 661-676, 2020.

[2] G. Dai and R. Hao, "Existence of solutions for a $p(x)$ Kirchhoff-type equation," Journal of Mathematical Analysis and Applications, vol. 359, no. 1, pp. 275-284, 2009.

[3] N. T. Chung, "Infinitely many solutions for some fourth order elliptic equations of $p(x)$-Kirchhoff type," in Differential Equations and Dynamical Systems, pp. 1-15, Springer, Berlin, Germany, 2020.

[4] Q. Zhang and C. Zhao, "Existence of strong solutions of ap $(x)$ Laplacian Dirichlet problem without the Ambrosetti-Rabinowitz condition," Computers \& Mathematics with Applications, vol. 69, no. 1, pp. 1-12, 2015.

[5] N. T. Chung, "On the existence of solutions for a class of fourth order elliptic equations of Kirchhoff type with variable exponent," Advances in the Theory of Nonlinear Analysis and Its Application, vol. 3, no. 1, pp. 35-45, 2019.

[6] N. T. Chung, "Multiple solutions for a fourth-order elliptic equation of Kirchhoff type with variable exponent," AsianEuropean Journal of Mathematics, vol. 13, pp. 289-303, 2020. 
[7] F. Li and R. H. Liu, "The application of $p(x)$ harmonic mapping in image processing," Journal of Image and Graphics, vol. 13, pp. 19-23, 2008.

[8] Y. Chen, S. Levine, and M. Rao, "Variable exponent, linear growth functionals in image restoration," SIAM Journal on Applied Mathematics, vol. 66, no. 4, pp. 1383-1406, 2006.

[9] J. L. Lions, "On some questions in boundary value problems of mathematical physics," Contemporary Developments in Continuum Mechanics and Partial Differential Equations, vol. 30, pp. 284-346, 1978.

[10] A. Arosio and S. Panizzi, "On the well-posedness of the Kirchhoff string," Transactions of the American Mathematical Society, vol. 348, no. 1, pp. 305-330, 1996.

[11] F. J. S. A. Corrêa and R. G. Nascimento, "On a nonlocal elliptic system of p-Kirchhoff-type under Neumann boundary condition," Mathematical and Computer Modelling, vol. 49, pp. 598-604, 2008.

[12] X. He and W. Zou, "Infinitely many positive solutions for Kirchhoff-type problems," Nonlinear Analysis: Theory, Methods \& Applications, vol. 70, no. 3, pp. 1407-1414, 2009.

[13] G. Dai and D. Liu, "Infinitely many positive solutions for a $\mathrm{p}(\mathrm{x})$-Kirchhoff-type equation," Journal of Mathematical Analysis and Applications, vol. 359, no. 2, pp. 704-710, 2009.

[14] A. Ambrosetti and P. H. Rabinowitz, "Dual variational methods in critical point theory and applications," Journal of Functional Analysis, vol. 14, no. 4, pp. 349-381, 1973.

[15] J. Yao, "Solutions for Neumann boundary value problems involving -Laplace operators," Nonlinear Analysis: Theory, Methods \& Applications, vol. 68, no. 5, pp. 1271-1283, 2008.

[16] A. E. Amrouss, F. Moradi, and M. Moussoui, "Existence of solutions for fourth-order PDEs with variable exponents," The Electronic Journal of Differential Equations, vol. 153, pp. 1-13, 2009.

[17] E. Guo and P. Zhao, "Existence and multiplicity of solutions for nonlocal $\mathrm{p}(\mathrm{x})$-Laplacian equations with nonlinear Neumann boundary conditions," Boundary Value Problems, vol. 2012, no. 1, pp. 1-15, 2012.

[18] N. T. Chung, "Existence of solutions for perturbed fourth order elliptic equations with variable exponents," Electronic Journal of Qualitative Theory of Differential Equations, vol. 96, no. 96, pp. 1-19, 2018

[19] X. B. Shu, Y. Z. Lai, and F. Xu, "Existence of infinitely many periodic subharmonic solutions for nonlinear non-autonomous neutral differential equations," The Electronic Journal of Differential Equations, vol. 2013, pp. 1-21, 2013.

[20] X.-B. Shu, Y. Lai, and F. Xu, "Existence of subharmonic periodic solutions to a class of second-order non-autonomous neutral functional differential equations," Abstract and Applied Analysis, vol. 2012, Article ID 404928, 26 pages, 2012.

[21] F. L. Wang and Y. K. An, "Existence and multiplicity of solutions for a fourth-order elliptic equation," Boundary Value Problems, vol. 2012, pp. 1-6, 2012.

[22] S. G. Zhang, "Multiplicity of solutions for Kirchhoff type equation involving the $p(x)$ - biharnonic operator," Journal of Shandong University (Natural Science), vol. 51, pp. 48-53, 2016.

[23] S. G. Zhang, "Multiple solutions of Navier boundary value problem for fourth-order elliptic equation with variable exponents," Journal of Shandong University (Natural Science), vol. 53, pp. 32-37, 2018.

[24] X. Fan and D. Zhao, "On the Spaces $\operatorname{Lp}(x)(\Omega)$ and Wm, $p(x)(\Omega)$," Journal of Mathematical Analysis and Applications, vol. 263, no. 2, pp. 424-446, 2001.
[25] A. B. Zang and Y. Fu, "Interpolation inequalities for derivatives in variable exponent lebesgue-sobolev spaces," Nonlinear Analysis: Theory, Methods \& Applications, vol. 69, pp. 3629-3636, 2007.

[26] M. Willem, Minimax Theorem, Birkhauser Verlag, Basel, Switzerland, 1996. 